Behav Immun, 1992, 6: 18 31

4 萨姆布鲁克 J, 弗里奇 E F, 曼尼阿蒂斯 T, 著. 分子克隆实验指南, 第 2 版. 北京: 科学出版社, 1992.28

5 Joycelyn E, Zhang S, Yang B H, et al. Characterization of the murine gene encoding the hyaluronan receptor RHAMM. Gene, 1995, 163: $233 \sim 238$

6 Jain R, Zwickler D. Corticotropirr releasing factor modulates the immune response to stress in the rat. Endocrinology, 1991, 128(3): 1329 1335

\title{
$\mathrm{Mg}^{2+}$ 诱导类囊体膜中 $\mathbf{L H C}$ 互的聚集 \\ 温晓刚 林世青* 匡廷云 \\ (中国科学院植物研究所, 北京 100093。* 联系人)
}

摘要 用高斯解叠程序分析了 $\mathrm{Mg}^{2+}$ 诱导小麦类囊体膜低温 $(77 \mathrm{~K})$ 荧光光谱的变化. 所有光谱 均可用 7 个高斯亚带进行拟合. $\mathrm{Mg}^{2+}$ 可以使 LHC 导多聚体 $\left(\mathrm{F}_{699}\right)$ 和 PS E $\left(\mathrm{F}_{685}\right.$ 和 $\left.\mathrm{F}_{695}\right)$ 的亚带面 积增加, 并使 LHC 宅三聚体 $\left(\mathrm{F}_{681}\right)$ 和 PS iv $\left(\mathrm{F}_{729}\right.$ 和 $\left.\mathrm{F}_{740}\right)$ 的亚带面积减少, 并且发现 $\mathrm{Mg}^{2+}$ 可使 $\mathrm{F}_{699}$ 对 $\mathrm{F}_{681}$ 的亚带面积比增加 1.72 倍. 由此可以得出结论, $\mathrm{Mg}^{2+}$ 可使类囊体膜中的 LHC 导三聚体 大量地聚集为多聚体。最后, 讨论了 LHC 宅聚集的可能含义.

\section{关键词 $\mathrm{LHC}$ 聚集 $\mathrm{Mg}^{2+}$ 荧光光谱 解叠}

高等植物光系统 (E) PS 司) 捕光叶绿素 $\mathrm{a} / \mathrm{b}$ 蛋白复合物系由一组十分相似的 LHC Ea, LHC E $\mathrm{b}$, LHC E $\mathrm{e}$ 和 LHC Ed 色素蛋白复合物所组成, 其中 LHC E $\mathrm{Eb}$ 是 PS E的主要外周天线蛋白复

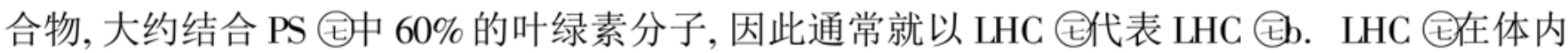
主要以三聚体状态存在 ${ }^{1]}$, 但在一定条件下 (如质子梯度和体内 $\mathrm{CO}_{2}$ 饥饿), LHC 宅还可部分聚 集为具有有序的三维空间的多聚体结构 ${ }^{[2,3]}$. 最近 Horton 等人 ${ }^{[4]}$ 建议, LHC 昰聚集态的变化 可能在光合激发能的分配调节, 特别是多余能量的耗散中起着十分重要的作用. 因此, 目前 LHC 毛聚集态的研究已成为光合作用的一个热点, 并引起光物理和光化学学家的广泛兴趣.

人们早就知道, $\mathrm{Mg}^{2+}$ 可以诱导体外提纯的 LHC 毛产生某种聚集反应, 并使含 LHC Є的人 工脂质体膜之间发生粘连 ${ }^{[5]}$, 但关于 $\mathrm{Mg}^{2+}$ 能否诱导类囊体膜中的 LHC 氜从三聚体聚集为多聚 体还未见到确切的证据. 本文通过高斯解叠分析, 定量地计算了加 $\mathrm{Mg}^{2+}$ 后类囊体膜低温荧光 光谱中各亚带荧光面积的变化, 特别是着重分析了 LHC E已聚体和多聚体的亚带荧光面积的 变化, 从而证明 $\mathrm{Mg}^{2+}$ 可诱导类囊体膜中的 LHC 氖从三聚体聚集为多聚体.

\section{1 材料与方法}

郑州 891 小麦( Triticum aestivum L. ) 在人工培养箱 $15{ }^{\circ} \mathrm{C}$ 恒温下用 Hoagland 溶液培养 $12 \mathrm{~d}$ 后, 取小麦幼苗叶片为材料, 按 $\operatorname{Lin}$ 等人 ${ }^{[6]}$ 的方法制备不含 $\mathrm{Mg}^{2+}$ 等多价阳离子的小麦类囊体 膜. 样品中叶绿素最终浓度为 $10 \mu \mathrm{g} \cdot \mathrm{mL}^{-1}$. 叶绿素含量按 $\operatorname{Arnon}^{[7]}$ 的方法测定. 类囊体膜的 低温 $(77 \mathrm{~K}$ ) 荧光发射光谱用日立 $\mathrm{F}-4500$ 荧光分光光度计测定, 荧光激发波长为 $436 \mathrm{~nm}$ ( 狭缝 5 $\mathrm{nm}$ ), 荧光发射波长为 $650 \sim 780 \mathrm{~nm}$ ( 狭缝 $2.5 \mathrm{~nm}$ ). 用微机程序对荧光光谱数据进行高斯解叠 和亚带苂光峰面积的计算. 


\section{2 结果与讨论}

已知体外 LHC 氖聚体的低温 $(77 \mathrm{~K})$ 苂光峰在 $681 \mathrm{~nm}\left(\mathrm{~F}_{681}\right)$, 而 LHC 导多聚体在 $681 \mathrm{~nm}$ 的 低温荧光峰强度大约只有三聚体的 $1 / 5$, 即发生了严重的荧光猝灭, 并在 $699 \mathrm{~nm}$ 左右出现 1 个 新的特征峰 $\left(\mathrm{F}_{699}\right)^{[3]}$. Murata ${ }^{[8]}$ 早已发现 $\mathrm{Mg}^{2+}$ 可以诱导类囊体膜低温苂光发射光谱发生显著 变化, 并正确地把它归于激发能在两个光系统之间的重新分配. 最近我们注意到, $\mathrm{Mg}^{2+}$ 诱导 小麦类囊体膜低温菼光差异光谱在 699nm 附近出现 1 个肩峰( 未显示)。由于 LHC 氖聚体和 多聚体的荧光峰 $\left(\mathrm{F}_{681}\right.$ 和 $\left.\mathrm{F}_{699}\right)$ 与 $P S$ 无的苂光峰 $\left(\mathrm{F}_{685}\right.$ 和 $\left.\mathrm{F}_{695}\right)$ 几乎完全相互重叠, 同时 $\mathrm{Mg}^{2+}$ 诱导 类囊体膜中 PS 毛的苂光强度变化又大大超过由于 LHC 毛聚集态的改变所引起的苂光强度变 化, 因此, 为了进一步证实 $\mathrm{Mg}^{2+}$ 是否对类囊体膜中 LHC 导聚集态的改变起作用, 我们用计算机 程序对苂光光谱曲线数据以不同高斯亚带进行解叠计算和分析. 图 1 是不含 $\mathrm{Mg}^{2+}$ 和含有 $\mathrm{Mg}^{2+}$ 的小麦类囊体膜低温荧光光谱高斯解叠的典型图. 结果发现, 所有光谱均可用以下 7 个 高斯亚带进行解叠拟合: $F_{685}$ 和 $F_{695}$ (PS 氞), $F_{729}$ 和 $F_{740}$ (PS iv), $F_{738}$ ( 振动带), 以及 $F_{681}$ ( LHC 氖三 聚体) 和 $\mathrm{F}_{699}$ ( LHC 氞多聚体) (图 1). 与 Siffel 和 $V_{a c h a}{ }^{[2]}$ 以烟草为材料相比, 少 1 个可能来自振 动带的 $F_{712}$ 亚带成分.
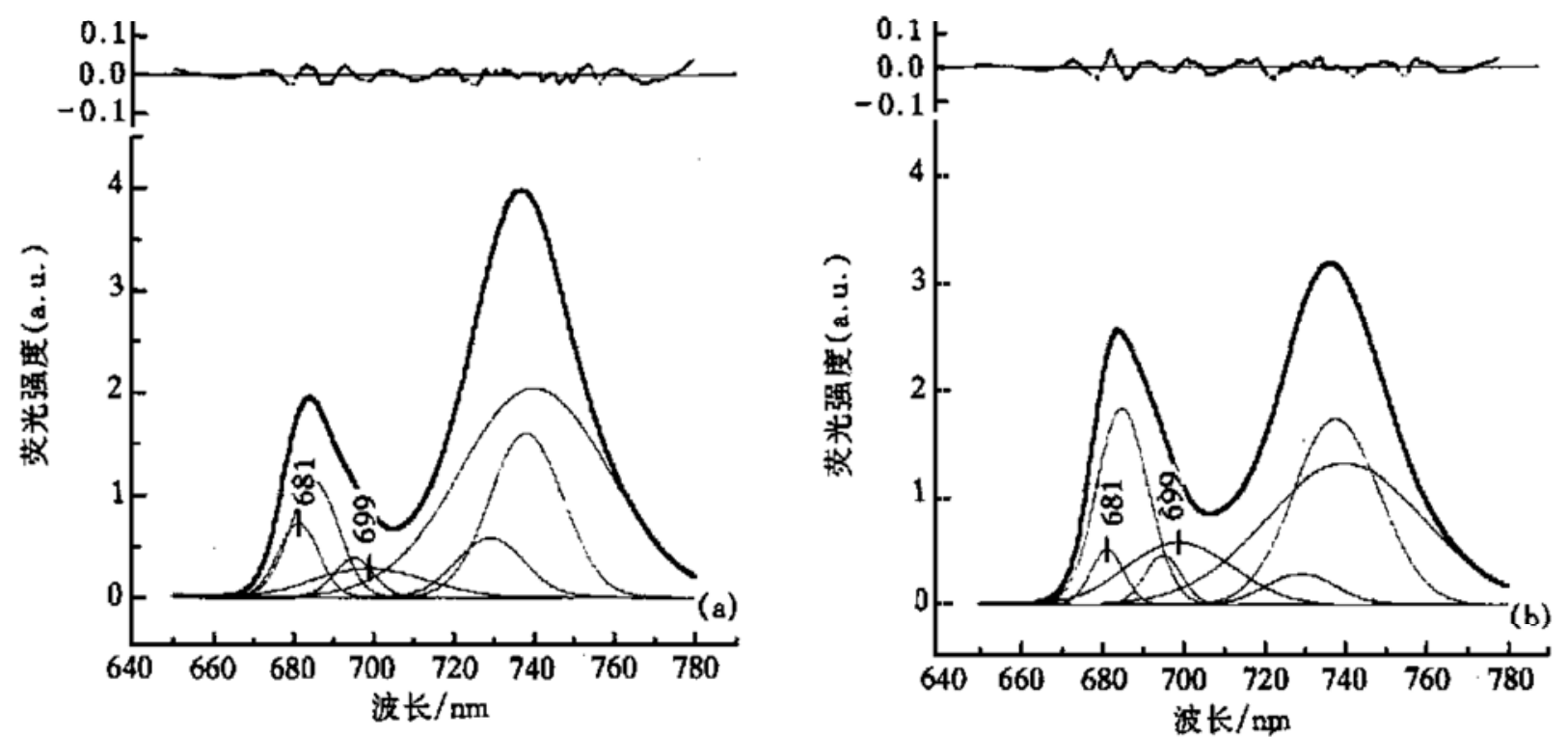

图 $1 \mathrm{Mg}^{2+}$ 诱导小麦类囊体膜低温 $(77 \mathrm{~K}$ ) 荧光光谱高斯解叠的亚带变化 (a) 不含 $\mathrm{MgCl}_{2}$; (b) 含有 $10 \mathrm{mmol} \cdot \mathrm{L}^{-1} \mathrm{MgCl}_{2}$. 上方小图曲线为解叠的误差

表 1 列出从 5 次独立实验得到的低温苂光光谱的高斯亚带解叠拟合的平均值及其误差. 结果表明, 加 $\mathrm{Mg}^{2+}$ 后 PS 宅的 $\mathrm{F}_{685}$ 和 $\mathrm{F}_{695}$ 亚带荧光面积分别比原来增加了 $62.3 \%$ 和 $35.3 \%$, 而 PS iv的 $\mathrm{F}_{729}$ 和 $\mathrm{F}_{740}$ 亚带苂光面积分别降低了 $50.5 \%$ 和 $19.7 \%$ 。这定量说明了 $\mathrm{Mg}^{2+}$ 增加激发能 向 PS E分配和减少向 PS iv分配的程度, 以及激发能在一些叶绿素蛋白复合体之间重新分配的

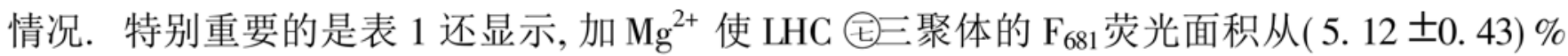
降低为 $(3.38 \pm 0.69) \%$, 下降了 34.0\% ; LHC 氖多聚体的 $\mathrm{F}_{699}$ 荧光面积从 $(5.23 \pm 1.17) \%$ 增加 为( 9.37 土1. 44) \%, 增加了 $79.2 \%$, 结果导致 LHC Є多聚体对三聚体的比值 $\left(\mathrm{F}_{699} / \mathrm{F}_{681}\right)$ 从加 $\mathrm{Mg}^{2+}$ 前的 1.02 增加为加 $\mathrm{Mg}^{2+}$ 后的 2. 77, 即增加了 1.72 倍. 上述结果清楚地说明, $\mathrm{Mg}^{2+}$ 的确 
可诱导类囊体膜中 LHC E从三聚体大量地聚集为多聚体. 我们对室温下 LHC 宅在类囊体膜中 聚集动力学的进一步研究表明, $\mathrm{Mg}^{2+}$ 诱导 LHC 毛的聚集作用是以两种不同速率进行的(未发 表), 此时它们正以两种不同速度从基质向基粒膜区迁移 ${ }^{[6]}$.

表 1 小麦类囊体膜低温 (77K) 苂光光谱高斯解叠的亚带荧光面积a)

\begin{tabular}{|c|c|c|c|}
\hline \multirow{2}{*}{ 波长/nm } & \multicolumn{2}{|c|}{ 亚带苂光面积/ \% b) } & \multirow{2}{*}{$\begin{array}{c}\text { 亚带苂光面积比 } / \% \\
\left(\text { 含有 } \mathrm{Mg}^{2+} \text { / 不含 } \mathrm{Mg}^{2+} \text { ) }\right.\end{array}$} \\
\hline & 不含 $\mathrm{Mg}^{2+}$ & 含有 $\left.\mathrm{Mg}^{2+} \mathrm{c}\right)$ & \\
\hline 681 & $5.12 \pm 0.43$ & $3.38 \pm 0.69$ & 66.0 \\
\hline 685 & $10.56 \pm 1.62$ & 17. $10 \pm 2.01$ & 162.3 \\
\hline 695 & $2.24 \pm 0.61$ & $3.03 \pm 0.48$ & 135.3 \\
\hline 699 & $5.23 \pm 1.17$ & $9.37 \pm 1.44$ & 179. 2 \\
\hline 729 & $9.33 \pm 3.03$ & 4. $62 \pm 2.52$ & 49.5 \\
\hline 738 & $18.82 \pm 4.44$ & $23.35 \pm 6.34$ & 124. 1 \\
\hline 740 & $48.75 \pm 2.33$ & $39.15 \pm 5.33$ & 80.3 \\
\hline
\end{tabular}

a) 所有数据均为 5 次独立试验的平均值 土标准差 $(n=5)$; b) 亚带荧光面积以占总荧光光谱面积百分比表示; c) $\mathrm{MgCl}_{2}$ 浓度为 $10 \mathrm{mmol} \cdot \mathrm{L}^{-1}$

Garab 等人 ${ }^{[9]}$ 用 $\mathrm{CD}$ 光谱技术推测, LHC 毛在类囊体膜中可能会形成有利于激发能长距离 传递和调控的三维空间的网络结构. Ruban 等人 ${ }^{[3]}$ 用 LD 光谱等方法证明类囊体膜中部分 LHC 国是以多聚体形式存在, 其聚集程度受类囊体膜的 $\mathrm{pH}$ 梯度所控制. 我们的工作表明, $\mathrm{Mg}^{2+}$ 浓度的变化也可控制类囊体膜中 LHC 毛的聚集. 考虑到在光照情况下, 类囊体膜的内腔 在产生酸化的同时还发生 $\mathrm{Mg}^{2+}$ 外流, 导致膜外基质部分空间中的 $\mathrm{Mg}^{2+}$ 浓度增加等变化, 最 近, Horton 等人 ${ }^{[4]}$ 推测, 这些类囊体膜微环境的变化必然引起 LHC 导聚集态的改变, 从而对激 发能的利用和耗散产生影响. 植物如何能在有限的光照下高效地利用激发能, 又能在过量的 光照下有效地耗散多余的激发能,这一直是人们十分感兴趣的问题. 这个问题的解决还有赖 于人们对 LHC 导多聚体的性质及 LHC 导在体内聚集态的调控机制做进一步的研究.

致谢 本工作为国家自然科学基金(批准号: 39670068, 39800086) 资助项目.

\section{参 考 文 献}

$1 \mathrm{~K} \cdot$ hlbrandt W, Wang D N, Fujiyoshi Y. Atomic model of plant light-harvesting complex by electron crystallography. Nature, 1994, 367: 614 621

2 Siffel P, Vacha F. LHC aggregation in intact leaves of tobacco plants stressed by $\mathrm{CO}_{2}$ starvation. In: Mathis $\mathrm{P}$ ed. Photosynthesis: from Light to Biosphere. Montpellier, 1995. Dordrecht: Kluwer Academic Publishers, 1995. Vol 1. 111 114

3 Ruban A V, Calkoen F, Kwa S L S, et al. Characterization of LHC () in the aggregated state by linear and circular dichroism spectroscopy. Biochim Biophys Acta, 1997, 1321: 61 70

4 Horton P, Ruban A V, Walters R G. Regulation of light harvesting in green plants. Annu Rev Plant Physiol Plant Mol Biol, 1996, 47: $655 \sim 684$

5 Mullet J E, Arntzen C J. Simulation of grana stacking in a model membrane system mediation by a purified light-harvesting pigment-protein complex from chloroplasts. Biochim Biophys Acta, 1980, 589: 100 117

6 Lin S Q, Xu L, Kuang T Y, et al. A new type of the heterogeneity of the photosystem (). In: Murata N ed. Research in Photosynthesis, Nagoya, 1992. The Netherlands: Kluwer Academic Publishers, 1992, Vol 2, 769 772

7 Arnon D N. Copper enzymes in isolated chloroplasts. Plant Physiol, 1949, 24(1) : 1 15

8 Murata N. Control of excitation transport in photosynthesis (). Magnesium ion_dependent distribution of excitation energy between two 
pigment systems in spinach chloroplasts. Biochim Biophys Acta, 1969, 189: 171 181

9 Garab G, Kieleczawa J, Sutherland J C, et al. Organisation of pigment-protein complexes into macrodomains in the thylakoid membranes of wild type and chlorophyll brless mutant of barley as revealed by circular dichroism. Photochem Photobiol, 1991, 54: 273 281

\title{
一种简单有效的检测基因表达产物的方法
}

\author{
许家喜 麻 密 $^{(4)}$ 麻 远 $^{\left({ }^{(M)}\right.}$ 林忠平 $^{(4)}$
}

( 北京大学化学与分子工程学院, 北京 100871; (4中国科学院植物研究所, 北京 100093; (四清华大学化学系, 北京 100084)

摘要 外源基因转入动植物细胞后在翻译水平上正确表达产物的定性定量测定是基因工程中 的重要问题。通常生物中某些酶的含量很低, 如异戊烯基转移酶, 在进行其转基因研究时较难 测定. 根据其 cDNA 序列, 预测出抗原位点肽并用固相法合成, 与载体蛋白偶联后获得对其起 专一性反应的抗体, 用该抗体通过 ELISA 法可以有效测定转基因烟草中的基因表达产物. 为 测定难以分离纯化的低含量的基因表达产物提供了一种简便而有效的方法.

\section{关键词 转基因植物 异戊烯基转移酶 抗原肽 检测方法}

外源基因转入动植物细胞后是否能够在翻译水平上正确表达, 表达的量如何, 是基因工程 中至关重要的问题. 翻译水平上的检测, 虽然可以通过 Western blot 和 ELISA 等方法进行定性 和定量分析 ${ }^{[1]}$, 但对于一些编码生物体内原本含量很微的酶的基因而言, 获得它们的纯化的表 达产物十分困难. 本文报道一种在计算机辅助下根据 cDNA 序列预测出其抗原位点肽, 用固 相法合成后制成复合抗原, 免疫家兔后获得专一性抗体, 再通过 ELISA 和 Western blot 法测定 转基因植物中外源基因表达产物的一种简便而有效的方法.

\section{1 材料与方法}

（1）试剂和材料. 保护氨基酸 Boc-His (Tos), Boc-Arg(Tos), Boc-Ile, Boc-Ala, Boc-Glu (Obzl), Boc-Lys (Z), Boc-Phe, Boc-Gln, 氯甲基树脂(含氯量 $1.04 \mathrm{mmol} / \mathrm{g}, 1 \%$ 交联度), HOBt, HOSu 和三氟乙酸( Sigma 公司), Sephadex G15 (Pharmacia 公司), 辣根过氧化物酶标记的羊抗兔 IgG (HRP-羊抗兔 IgG) (华美公司), 其他试剂为国产分析纯. 烟草(Nicotina tobacco W38) 无菌 苗继代培养于 MS 培养基中, 取其叶片和茎段作为转基因受体而得到的转基因植株.

(2) 仪器. IBM PC586 计算机; 手工固相多肽合成仪; LKB2151 高压液相色谱仪, 柱 XWGC18 (4. $6 \mathrm{~mm}$ ( I. D. ) × $250 \mathrm{~mm}$ ) ; Hitachi 835-50 型氨基酸分析仪; Perkin-Elmer $241 \mathrm{MC}$ 旋光仪; ELISA Reader G4500 Bio-Rad 酶标仪. 核酸蛋白紫外检测仪, Bruker Instrum Biflex 四质谱仪.

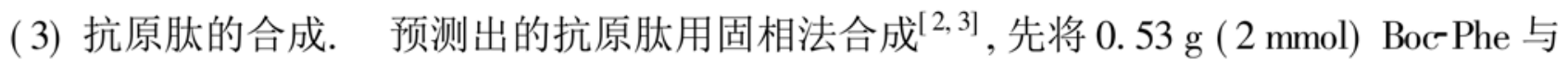
$0.33 \mathrm{~g}$ ( $1 \mathrm{mmol}$ ) 碳酸铯反应制成铯盐, 再连到 $0.5 \mathrm{~g}$ (含氯 $0.52 \mathrm{mmol}$ ) 固相载体氯甲基树脂上, 用无水乙酸钾、苯甲酰氯依次封闭, $30 \%$ 三氟乙酸/ 二氯甲烷脱保护, 三乙胺中和, 狮三酮法测 得氨基含量为 $0.35 \mathrm{mmol}(0.70 \mathrm{mmol} / \mathrm{g})$, 按预测出的抗原肽序列, 以 $1 \mathrm{mmol}$ 保护氨基酸为原 料, HOBt 或 HOSu 和 DCC 为缩合剂, 用逐步接长法依次接至所需肽段. 合成好的肽段以苯甲 醚和苯甲硫醚为捕获剂, 用无水氟化氢从树脂上切下及脱去侧链保护, 抽干氟化氢后, 冷无水 